\title{
In situ transmission electron microscopy of magnetic transitions
}

\author{
Andras Kovacs ${ }^{1}$, Laura Lewis ${ }^{2}$, Michalis Charilaou ${ }^{3}, \mathrm{~S} \mathrm{Guo}^{4}$ and Rafal Dunin-Borkowski ${ }^{5}$
}

${ }^{1}$ Ernst Ruska Centre, Forschungszentrum Juelich, United States, ${ }^{2}$ Northeastern University, United States, ${ }^{3}$ University of Louisiana at Lafayette, United States, ${ }^{4}$ Forschungszentrum Juelich, United States, ${ }^{5}$ Forchungszentrum Jülich, Nordrhein-Westfalen, Germany

Magnetic materials show a strong dependence of temperature that accompanies with disappearance of magnetic order at the Curie temperature and in some transition metal oxides, a phase transition that associated with major changes in conductivity and magnetic properties. Here we study the lowtemperature Verwey transition in single crystalline $\mathrm{Fe}_{3} \mathrm{O}_{4}$ and the high-temperature magnetic transition in a metallic Fe-Ni meteorite using various in situ transmission electron microscopy (TEM) methods supported by bulk measurements and micromagnetic simulations. Conventional imaging, electron diffraction, Lorentz microscopy and off-axis electron holography are used to reveal the structural and magnetic processes occurring in the transitions.

TEM specimens were prepared using focused Ga ion beam milling in a dual beam scanning electron microscope (ThermoFisher Helios 400). Surface damage was reduced by Ar ion sputtering at low energies in a Fischione Nanomill system. Figure 1 shows the TEM images of Verwey transition in thin film of a single crystalline $\mathrm{Fe}_{3} \mathrm{O}_{4}$ viewed along the [110] crystallographic direction. Conventional TEM images and electron diffraction patterns were recorded using a liquid nitrogen cooled double-tilt TEM holder (Gatan 636). The cubic to monoclinic phase transition was accompanied with appearance of deformation twins that formed a quickly passing reaction front in the sample as the temperature decreased (Fig. 1a-d) from 120 to $100 \mathrm{~K}$. The appearance of weak reflections in the electron diffraction patterns (Fig. 1e-h) confirmed the monoclinic phase formation. The corresponding magnetic state change was studied using Fresnel images and off-axis electron holograms recorded in magnetic field-free conditions (Lorentz mode) and a direct electron counting detector (Gatan/Ametek K2 IS) using an image aberration-corrected microscope (ThermoFisher 60-300). Fresnel imaging and electron holography were used to map the magnetic state changes during the phase transition by studying the magnetic domains and domain walls.

The NW6259 metallic meteorite (Fig. 2) contains several different Fe-Ni phases forming a magnetic composite with strong interphase magnetic coupling. The main phase is the chemically ordered $\mathrm{L} 1_{0}-\mathrm{FeNi}$ (tetrataenite) (Fig. 2b), which contains nanometer-sized Ni-poor precipitates and Ni-rich lamellae (Fig. 2d). The magnetic imaging studies revealed a complex magnetic domain structure (Fig. 2c) that was also confirmed by micromagnetic simulations. The bulk thermomagnetic measurements indicated a magnetic transition at $830 \mathrm{~K}$ in the pristine sample, which dropped to $740 \mathrm{~K}$ in the consecutive measurements. Annealing studies were carried out using a double tilt MEMS holder (DENSsolution). In situ annealing studies in the TEM carried out in both conventional and Lorentz mode to reveal the origin of the transition temperature variation and the role of constituent Fe-Ni phases in the magnetic state. 


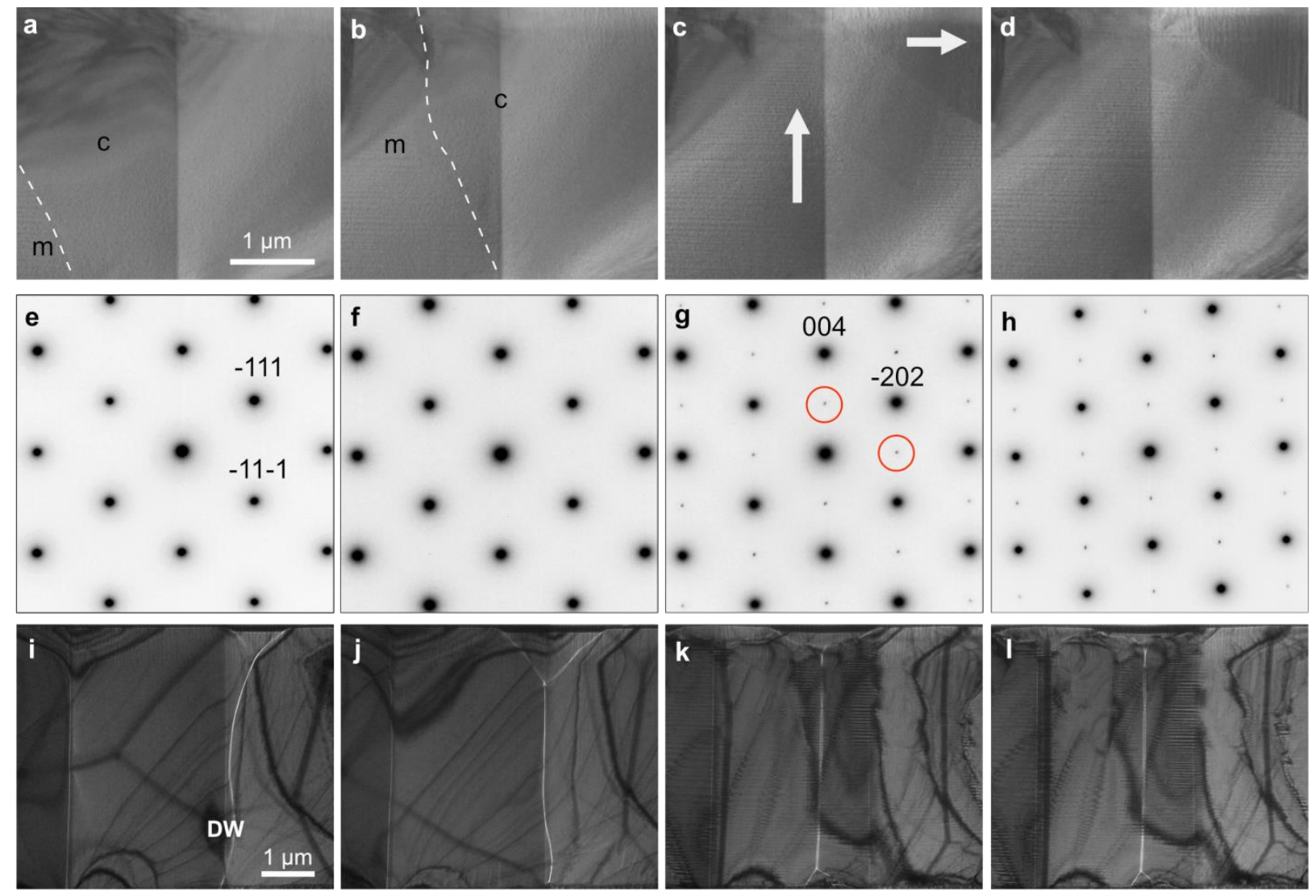

Figure 1. Verwey transition from cubic (c) to monoclinic (m) [110]-Fe3O4 presented in (a-d) BF TEM images, (e-h) electron diffraction patterns and (i-l) Fresnel defocused images. Arrows in (c) mark the caxis orientation of $\mathrm{m}-\mathrm{Fe} 3 \mathrm{O} 4$. In $(\mathrm{g})$, selected reflections indicate the $\mathrm{m}-\mathrm{Fe} 3 \mathrm{O} 4$ phase formation. In (i), DW mark a magnetic domain wall. Fresnel images were recorded with $0.5 \mathrm{~mm}$ defocus.
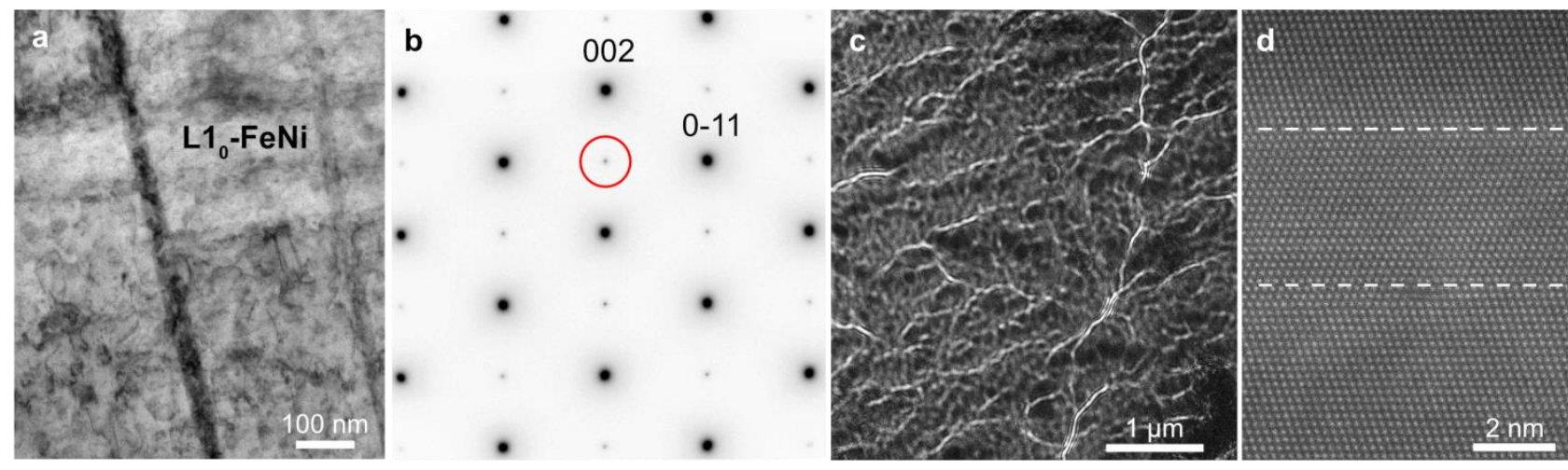

Figure 2. The structure of the slowly cooled NWA6259 metallic meteorite. (a) Brigh field scanning TEM (STEM) image shows lamellar precipitates in L10-FeNi host matrix. (b) Electron diffraction pattern confirms the chemical ordering. (c) Fresnel image recorded in magnetic field free conditions reveals the presence of high-density domain walls. (d) High-resolution high-angle annular dark-field STEM image of the lamella precipitate in $\mathrm{L} 10-\mathrm{FeNi}$. 


\section{Reference}

[1] This project has received funding from the ERC under the EU's Horizon 2020 research and innovation programme (Grant No. 856538, project "3D MAGiC") and from the German Research Foundation (DFG) via CRC/TRR 270 HoMMage (Project-ID 405553726). 